\title{
Some more results on $i$-convergence of filters
}

Rohini Jamwal, Shivani Sharma and Dalip Singh Jamwal

Department of Mathematics, University of Jammu, Jammu, India

Received: 5 January 2017, Accepted: 7 February 2017

Published online: 9 February 2017.

\begin{abstract}
In this paper, we have proved some more results on $I$-convergence of filters. We have proved the equivalence of $I$-convergence and ordinary convergence of filters as well as the equivalence of $I$-convergence of nets and filters.
\end{abstract}

Keywords: $I$-convergence of filters, equivalence of $I$-convergence,ordinary convergence of filters.

\section{Introduction}

The concept of convergence of a sequence of real numbers has been extended to statistical convergence independently by H. Fast [4] and I. J. Schoenberg [21]. Any convergent sequence is statistically convergent but the converse is not true [18]. Moreover, a statistically convergent sequence need not even be bounded [18]. Let $\mathbb{N}$ denotes the set of natural numbers. If $K \subset \mathbb{N}$, then $K_{n}$ will denote the set $\{k \in K: k \leq n\}$ and $\left|K_{n}\right|$ stands for the cardinality of $K_{n}$. The natural density of $K$ is defined by

$$
d(K)=\lim _{n} \frac{\left|K_{n}\right|}{n}
$$

if the limit exists [5,17].

The concept of $I$-convergence of real sequences $[7,8]$ is a generalization of statistical convergence which is based on the structure of the ideal $I$ of subsets of the set of natural numbers. The notion of ideal convergence for single sequences was first defined and studied by Kostyrko et. al. [7]. Mursaleen et. al. [13] defined and studied the notion of ideal convergence in random 2-normed spaces and construct some interesting examples. Several works on $I$-convergence and statistical convergence have been done in $[1,3,6,7,8,9,12,13,14,15,16,20]$.

The idea of $I$-convergence of real sequences coincides with the idea of ordinary convergence if $I$ is the ideal of all finite subsets of $\mathbb{N}$ and with the statistical convergence if $I$ is the ideal of subsets of $\mathbb{N}$ of natural density zero [10].

The idea of $I$-convergence has been extended from real number space to metric space [7] and to a normed linear space [19] in recent works. Later B. K. Lahiri and P. Das [10] extended the idea of $I$-convergence to an arbitrary topological space and observed that the basic properties are preserved in a topological space. In [11], they also introduced the idea of $I$-convergence of nets in a topological space and examined how far it affects the basic properties. [6] introduced the idea of $I$-convergence of filters in a topological space $X$ and studied its various properties. [6] proved that basic properties of convergence of filters in a topological space $X$ also hold in case of $I$-convergence of filters. We start with the following definitions.

Definition 1. Let $X$ be a non-empty set. Then a family $\mathscr{F} \subset 2^{X}$ is called a filter on $X$ if 
(i) $\emptyset \notin \mathscr{F}$,

(ii) $A, B \in \mathscr{F}$ implies $A \cap B \in \mathscr{F}$ and

(iii) $A \in \mathscr{F}, B \supset A$ implies $B \in \mathscr{F}$.

Definition 2. Let $X$ be a non-empty set. Then a family $I \subset 2^{X}$ is called an ideal of $X$ if

(i) $\emptyset \in I$,

(ii) $A, B \in I$ implies $A \cup B \in I$ and

(iii) $A \in I, B \subset A$ implies $B \in I$.

Definition 3. Let $X$ be a non-empty set. Then a filter $\mathscr{F}$ on $X$ is said to be non-trivial if $\mathscr{F} \neq\{X\}$.

Definition 4. Let $X$ be a non-empty set. Then an ideal I of $X$ is said to be non-trivial if $I \neq\{\emptyset\}$ and $X \notin I$.

Note (i) $\mathscr{F}=\mathscr{F}(I)=\{A \subset X: X \backslash A \in I\}$ is a filter on $X$, called the filter associated with the ideal $I$.

(ii) $I=I(\mathscr{F})=\{A \subset X: X \backslash A \in \mathscr{F}\}$ is an ideal of $X$, called the ideal associated with the filter $\mathscr{F}$.

(iii) A non-trivial ideal $I$ of $X$ is called admissible if $I$ contains all the singleton subsets of $X$.

Several examples of non-trivial admissible ideals have been considered in [7].

Throughout this paper, $X=(X, \tau)$ will stand for a topological space and $I=I(\mathscr{F})$ will be the ideal of $X$ associated with the filter $\mathscr{F}$ on $X$.

Before proving some more results on $I$-convergence of filters, we give a brief discussion on $I$-convergence of filters as given by [6].

Definition 5. A filter $\mathscr{F}$ on $X$ is said to be $I$-convergent to $x_{0} \in X$ if for each nbd $U$ of $x_{0},\{y \in X: y \notin U\} \in I$. In this case, $x_{0}$ is called an $I-\operatorname{limit}$ of $\mathscr{F}$ and is written as $I-\lim \mathscr{F}=x_{0}$.

Theorem 1. A filter $\mathscr{F}$ on $X$ is $I-$ convergent to $x_{0}$ if and only iffor each nbd $U$ of $x_{0},\{V \in \mathscr{P}(X): U \cap V=\emptyset\} \subset I$.

Proposition 1. If X is Hausdorff, then any I-convergent filter $\mathscr{F}$ on X has a unique I-limit.

Notation In case more than one filter is involved, we use the notation $I(\mathscr{F})$ to denote the ideal associated with the corresponding filter $\mathscr{F}$.

Proposition 2. Let $E \subset X$ and $\mathscr{F}$ be a filter on $E$ which is $I$-convergent to $x_{0} \in X$, where $I=I(\mathscr{F})$ is an admissible ideal of $E$. Then $x_{0}$ is a limit point of $E$. Conversely, if $x_{0}$ is a limit point of $E$, then there is a filter on $E \backslash\left\{x_{0}\right\}$ which is $I$-convergent to $x_{0}$, for some admissible ideal I of $E$.

Proposition 3. Let $X$ and $Y$ be two topological spaces and $f: X \rightarrow Y$ be a map. Let $\mathscr{F}$ be a filter on $X$. Then $f: X \rightarrow Y$ is continuous at $x_{0} \in X$ if and only if $I_{X}-\lim \mathscr{F}=x_{0}$ in $X$ implies $I_{Y}-\lim f(\mathscr{F})=f\left(x_{0}\right)$, where $I_{X}=I_{X}(\mathscr{F}), f(\mathscr{F})$ is a filter on $Y$ generated by the base $\{f(F): F \in \mathscr{F}\}$ and $I_{Y}=I_{Y}(f(\mathscr{F}))$.

\subsection{Characterization of closure}

Proposition 4. Let $E \subset X$. Then $x_{0} \in \bar{E}$ if and only if there is a filter $\mathscr{F}$ on $X$ such that $E \in \mathscr{F}$ and $I-\lim \mathscr{F}=x_{0}$.

Proposition 5. Let $\mathscr{F}$ be a filter on $X$ such that $I-\lim \mathscr{F}=x_{0}$. Then every filter $\mathscr{F}^{\prime}$ finer than $\mathscr{F}$ also $I-$ converges to $x_{0}$, where $I=I(\mathscr{F})$.

Remark. Let $\mathscr{F}$ be a filter on $X$ and $\mathscr{F}^{\prime}$ be another filter on $X$ finer than $\mathscr{F}$. Then $I\left(\mathscr{F}^{\prime}\right)-\lim \mathscr{F}^{\prime}=x_{0}$ need not imply that $I(\mathscr{F})-\lim \mathscr{F}=x_{0}$. 
Proposition 6. Let $\mathscr{F}$ be a filter on $X$ such that $I-\lim \mathscr{F}=x_{0}$. Then every filter $\mathscr{F}^{\prime}$ on $X$ coarser than $\mathscr{F}$ also $I$-converges to $x_{0}$, where $I=I(\mathscr{F})$.

Note The above proposition need not be true if we replace $I(\mathscr{F})-\lim \mathscr{F}^{\prime}$ by $I\left(\mathscr{F}^{\prime}\right)-\lim \mathscr{F}^{\prime}$.

Proposition 7.Let $\mathscr{F}$ be a filter on $X$ and $\mathscr{G}$ be any other filter on $X$ finer than $\mathscr{F}$. Then $I(\mathscr{F})-\lim \mathscr{G}=x_{0}$ implies $I(\mathscr{G})-\lim \mathscr{G}=x_{0}$. But not conversely.

Proposition 8.Let $\tau_{1}$ and $\tau_{2}$ be two topologies on $X$ such that $\tau_{1}$ is coarser than $\tau_{2}$. Let $\mathscr{F}$ be a filter on $X$ such that $I-\lim \mathscr{F}=x_{0}$ w.r.t $\tau_{2}$. Then $I-\lim \mathscr{F}=x_{0}$ w.r.t $\tau_{1}$. But the converse need not be true.

Proposition 9.Let $\mathscr{M}$ be a collection of all those filters $\mathscr{G}$ on a space $X$ which $I(\mathscr{G})$-converges to the same point $x_{0} \in X$. Then the intersection $\mathscr{F}$ of all the filters in $\mathscr{M} I(\mathscr{F})$-converges to $x_{0}$.

Proposition 10.If every I-convergent filter $\mathscr{F}$ on X has a unique I-limit, then the space X is Hausdorff.

Theorem 2.A filter $\mathscr{F} I_{X}$-converges to $x$ in $X=\prod_{\alpha \in \Lambda} X_{\alpha}$ if and only if $p_{\alpha}(\mathscr{F}) I_{X_{\alpha}}$-converges to $p_{\alpha}(x), \forall \alpha$, where $I_{X}=I_{X}(\mathscr{F})$ and $I_{X_{\alpha}}=I_{X_{\alpha}}\left(p_{\alpha}(\mathscr{F})\right)$.

This paper is an extension of the work done on $I$-convergence of filters in [6] and is inspired from [2,22].

\section{Equivalence of $I$-convergence and convergence of a filter $\mathscr{F}$}

We recall the following.

Let $\mathscr{F}$ be a filter on $X$ and let $\mathscr{D}$ be a set that is bijective with the filter $\mathscr{F}$. We shall call $\mathscr{D}$ an index set for $\mathscr{F}$ and denote the bijective correspondance by $\mathscr{F}=\left\{F_{d}: d \in \mathscr{D}\right\}$.

Note It is easy to show that $\mathscr{D}$ becomes a poset with the partial order defined by

$$
c \leq d \text { if and only if } F_{c} \supset F_{d} .
$$

In this case, we speak of an indexed filter.

Definition 6. Let $\mathscr{F}$ be an indexed filter on $X$ with index set $\mathscr{D}$. Any net $\lambda: \mathscr{D} \rightarrow X$ with $\lambda(d) \in F_{d}$ is called a derived net of $\mathscr{F}$.

Definition 7. Let $\lambda$ be a net in $X$ with directed set $\mathscr{D}$. Then $\mathscr{F}=\{F \subset X: \lambda$ is eventually in $F\}$ is called a derived filter of $\lambda$. By $\lambda$ eventually in $F$, we mean that some tail of $\lambda$ is contained in $F$. By tail of $\lambda$, we mean the set $\Lambda_{d}=\{\lambda(c): c \geq$ $d$ in $\mathscr{D}\}$.

Definition 8. $A$ net $\lambda: \mathscr{D} \rightarrow X$ in $X$ is said to be convergent to $x_{0} \in X$ if for each nbd $U$ of $x_{0}$, there is some $d \in \mathscr{D}$ such that $c \geq d$ in $\mathscr{D}$ implies that $\lambda(c) \in U$. In other words, some tail $\Lambda_{d}=\{\lambda(c): c \geq d$ in $\mathscr{D}\} \subset U$.

Theorem 3. A filter $\mathscr{F}$ on $X I$-converges to $x_{0} \in X$ if and only if every derived net $\lambda$ of $\mathscr{F}$ converges to $x_{0}$.

Proof. Suppose $I-\lim \mathscr{F}=x_{0}$. This means that for each nbd $U$ of $x_{0},\{V \in \mathscr{P}(X): U \cap V=\emptyset\} \subset I$. Let us index $\mathscr{F}$ with an index set $\mathscr{D}$ so that $\mathscr{F}=\left\{F_{s}: s \in \mathscr{D}\right\}$. Let us give some direction to $\mathscr{D}$ so that $c \geq d$ in $\mathscr{D}$ if and only if $F_{c} \subset F_{d}$. Let $\lambda$ be a derived net of $\mathscr{F}$ so obtained. We have to show that the net $\lambda$ converges to $x_{0}$. For this, let $U$ be a nbd of $x_{0}$. Since $U \cap(X \backslash U)=\emptyset$, we find that $X \backslash U \in I$. This implies that $U \in \mathscr{F}$. Now $U \in \mathscr{F}$ implies $U=F_{d}$, for some $d \in \mathscr{D}$. Now if $c \geq d$, then $F_{c} \subset F_{d}$ and so $\lambda(c) \in F_{c} \subset F_{d}=U$. Thus there is some tail $\Lambda_{d}=\{\lambda(c): c \geq d$ in $\mathscr{D}\}$ of $\lambda$ such that $\Lambda_{d} \subset U$. That is, $\lambda$ is eventually in $U$. Thus $\lambda \rightarrow x_{0}$. 
Conversely, suppose every derived net $\lambda$ converges to $x_{0}$. We shall show that $I-\lim \mathscr{F}=x_{0}$. For this, let $U$ be a nbd of $x_{0}$. We claim that $\{V \in \mathscr{P}(X): U \cap V=\emptyset\} \subset I$. Since $\lambda \rightarrow x_{0}$, tail $\Lambda_{d}=\{\lambda(c): c \geq d$ in $\mathscr{D}\} \subset U$. Since $\lambda$ is a derived net, there exists $F_{c} \in \mathscr{F}$ for $c \in \mathscr{D}$ such that $\lambda(c) \in F_{c}$. Thus $\Lambda_{d} \in \mathscr{F}$. Now, let $V \in \mathscr{P}(X)$ such that $U \cap V=\emptyset$. Then $V \subset X \backslash U$ implies $V \subset X \backslash \Lambda_{d}\left(\because \Lambda_{d} \subset U\right)$ and so $V \in I\left(\because \Lambda_{d} \in \mathscr{F}\right)$. Therefore, $\{V \in \mathscr{P}(X): U \cap V=\emptyset\} \subset I$. Hence $I-\lim \mathscr{F}=x_{0}$. This completes the proof.

We recall [22] that a filter $\mathscr{F}$ on a topological space $X$ is said to converge to $x_{0}$ (written as $\mathscr{F} \rightarrow x_{0}$ ) if $\mathscr{F}$ is finer than the nbd filter at $x_{0}$ (i.e., $\mathscr{U}_{x_{0}} \subset \mathscr{F}$ ). Using Theorem $2 \cdot 4$, we can prove the equivalence of $I$-convergence and convergence of a filter $\mathscr{F}$.

Theorem 4. A filter $\mathscr{F}$ on $X I$-converges to $x_{0} \in X$ if and only if $\mathscr{F}$ converges to $x_{0}$. Proof We know that a filter $\mathscr{F}$ converges to $x_{0}$ in a topological space $X$ if and only if every derived net $\lambda$ does [22]. Using Theorem $2 \cdot 4$, we get the required result.

Theorem 5. A net $\lambda: \mathscr{D} \rightarrow X$ converges to $x_{0} \in X$ if and only if the derived filter $\mathscr{F}$ of $\lambda I-$ converges to $x_{0}$. Proof Suppose a net $\lambda: \mathscr{D} \rightarrow X$ converges to $x_{0} \in X$. This means that for each nbd $U$ of $x_{0}$, some tail $\Lambda_{d}=\{\lambda(c): c \geq d$ in $\mathscr{D}\} \subset U$. Since $\mathscr{F}$ is a derived filter, each nbd $U \in \mathscr{F}$ (by definition of derived filter and the given condition). We have to show that $I-\lim \mathscr{F}=x_{0}$. For this, let $U$ be a nbd of $x_{0}$. We claim that $\{V \in \mathscr{P}(X): U \cap V=\emptyset\} \subset$ I. So, let $V \in \mathscr{P}(X)$ such that $U \cap V=\emptyset$. Now $U \cap V=\emptyset$ implies that $V \subset X \backslash U$. Since $U \in \mathscr{F}, X \backslash U \in I$. Further, $I$ is an ideal of $X$ and $V \subset X \backslash U$ implies that $V \in I$. Therefore, $\{V \in \mathscr{P}(X): U \cap V=\emptyset\} \subset I$. Conversely, suppose $\mathscr{F}$ is a derived filter of $\lambda$ such that $I-\lim \mathscr{F}=x_{0}$. This means that for each nbd $U$ of $x_{0},\{V \in \mathscr{P}(X): U \cap V=\emptyset\} \subset I \cdots(*)$. We shall show that the net $\lambda: \mathscr{D} \rightarrow X$ converges to $x_{0}$. Suppose $\lambda$ does not converge to $x_{0}$. This means that there is some nbd $U$ of $x_{0}$ such that $\lambda$ is not eventually in $U$. That is, $\Lambda_{d}=\{\lambda(c): c \geq d$ in $\mathscr{D}\} \nsubseteq U$, for any tail $\Lambda_{d}$. Since $\mathscr{F}$ is a derived filter, $U \notin \mathscr{F}$. From (*), $U \cap(X \backslash U)=\emptyset$ implies that $X \backslash U \in I$. This further implies that $U \in \mathscr{F}$, which is not true. Therefore, our supposition is wrong. Hence $\lambda \rightarrow x_{0}$.

\section{Equivalence of $I$-convergence of filters and nets}

We first define the $I$-convergence of nets in $X$.

Definition 9. Let I be a non-trivial ideal of subsets of $X$. Let $\lambda: \mathscr{D} \rightarrow X$ be a net in $X$, where $\mathscr{D}$ is a directed set. Then $\lambda$ is said to be $I$-convergent to $x_{0}$ in $X$ if for each nbd $U$ of $x_{0},\{\lambda(c) \in X: \lambda(c) \notin U\} \in I$.

Theorem 6. A filter $\mathscr{F}$ on $X I-$ converges to $x_{0} \in X$ if and only if every derived net $\lambda$ of $\mathscr{F} I$-converges to $x_{0}$, where $I=I(\mathscr{F})$.

Proof. Suppose $I-\lim \mathscr{F}=x_{0} \cdots(*)$. Let us index $\mathscr{F}$ with an index set $\mathscr{D}$ so that $\mathscr{F}=\left\{F_{d}: d \in \mathscr{D}\right\}$. Let us give some direction to $\mathscr{D}$ so that $c \geq d$ in $\mathscr{D}$ if and only if $F_{c} \subset F_{d}$. Let $\lambda: \mathscr{D} \rightarrow X$ be the derived net of $\mathscr{F}$ so obtained. This means that $\lambda(c) \in F_{c}$, for some $c \in \mathscr{D}$. We have to show that $I-\lim \lambda=x_{0}$. For this, let $U$ be a nbd of $x_{0}$. We claim that $\{\lambda(c) \in X: \lambda(c) \notin U\} \in I$. So, let $\lambda(c) \in X$ such that $\lambda(c) \notin U$. Then by the given condition $(*),\{\lambda(c)\} \in I$. Hence $\{\lambda(c) \in X: \lambda(c) \notin U\} \in I$.

Conversely, suppose every derived net $\lambda: \mathscr{D} \rightarrow X$ of $\mathscr{F} I$-converges to $x_{0} \cdots(* *)$. We have to show that $I-\lim \mathscr{F}=x_{0}$. For this, let $U$ be a nbd of $x_{0}$. We claim that $\{y \in X: y \notin U\} \in I$. So, let $y \in X$ such that $y \notin U$. We need to show that $\{y\} \in I$. If $y \neq \lambda(c)$, for any $c \in \mathscr{D}$, then clearly $y \notin F_{c}$, for any $c \in \mathscr{D}$ and so $y \in X \backslash F_{c}$, for some $c \in \mathscr{D}$. This implies that $\{y\} \in I$. If $y=\lambda(c)$, for some $c \in \mathscr{D}$, then $y \notin U$ implies that $\lambda(c) \notin U$. By the given condition $(* *),\{\lambda(c)\} \in I$. This implies that $\{y\} \in I$. 
Lemma 1. A filter $\mathscr{F}$ on $X$ converges to $x_{0}$ in $X$ if and only if every derived net $\lambda$ of $\mathscr{F} I$-converges to $x_{0}$. Proof It follows from Theorems $2 \cdot 5$ and $3 \cdot 2$.

Theorem 7. Let $\lambda: \mathscr{D} \rightarrow X$ be a net in $X$ and $\mathscr{F}$ be a derived filter of $\lambda$. Then $\lambda I-$ converges to $x_{0}$ in $X$ if and only if the derived filter $\mathscr{F} I$-converges to $x_{0}$, where $I=I(\mathscr{F})$.

Proof. Let $\lambda: \mathscr{D} \rightarrow X$ be a net in $X$ and $\mathscr{F}$ be a derived filter of $\lambda$. Suppose $I-\lim \lambda=x_{0}$. Then for each nbd $U$ of $x_{0}$, $\{\lambda(c) \in X: \lambda(c) \notin U\} \in I \cdots(*)$. We have to show that $I-\lim \mathscr{F}=x_{0}$. For this, let $U$ be a nbd of $x_{0}$. We claim that $\{y \in X: y \notin U\} \in I$. So, let $y \in X$ such that $y \notin U$. If $y=\lambda(c)$, for some $c \in \mathscr{D}$, then clearly by the given condition $\{y\} \in I$. If $y \neq \lambda(c)$, for any $c \in \mathscr{D}$, then we proceed as follows. $y \neq \lambda(c)$, for any $c \in \mathscr{D}$ implies that $y \notin \Lambda_{d}$, for any tail $\Lambda_{d}$ of $\lambda$. Thus $\Lambda_{d} \subset U$. Since $\mathscr{F}$ is a derived filter, by definition $U \in \mathscr{F}$. This implies that $X \backslash U \in I$. Now $y \notin U$ implies that $y \in X \backslash U$ and so $\{y\} \in I$. Therefore, $\{y \in X: y \notin U\} \in I$. Hence $I-\lim \mathscr{F}=x_{0}$.

Conversely, suppose that $I-\lim \mathscr{F}=x_{0}$. Then for each nbd $U$ of $x_{0},\{y \in X: y \notin U\} \in I \cdots(* *)$. We have to show that $I-\lim \lambda=x_{0}$. For this, let $U$ be a nbd of $x_{0}$. We claim that $\{\lambda(c) \in X: \lambda(c) \notin U\} \in I$. So, let $\lambda(c) \in X$ such that $\lambda(c) \notin U$. Clearly, by the given condition $(* *),\{\lambda(c)\} \in I$. Therefore, $I-\lim \lambda=x_{0}$.

We have the following definition of $I$-convergence of nets in $X$ as given by [11].

Definition 10. Let $\mathscr{D}$ be a directed set. Let I be a non-trivial ideal of subsets of $\mathscr{D}$. A net $\lambda: \mathscr{D} \rightarrow X$ is said to be $I$-convergent to $x_{0}$ in $X$ if for each nbd $U$ of $x_{0},\{c \in \mathscr{D}: \lambda(c) \notin U\} \in I$.

With the help of an example, we shall show that $I(\mathscr{F})-\lim \mathscr{F}=x_{0}$ need not imply that $I(\lambda)-\lim \lambda=x_{0}$, where $I(\mathscr{F})$ is the ideal associated with the filter $\mathscr{F}$ and $I(\lambda)$ is the non-trivial ideal of subsets of the directed set $\mathscr{D}$ of $\lambda$.

Example 1. Let $X=\{1,2,3\}$ with $\tau=\{\emptyset,\{1\}, X\}$. Here, $\mathscr{U}_{1}=\{\{1\},\{1,2\},\{1,3\}, X\}, \mathscr{U}_{2}=\{X\}$ and $\mathscr{U}_{3}=\{X\}$. Let $\mathscr{F}=\{\{1\},\{1,2\},\{1,3\}, X\}$. Then $I(\mathscr{F})=\{\emptyset,\{2\},\{3\},\{2,3\}\}$. We can easily see that 1,2 and 3 are $I(\mathscr{F})-$ limits of $\mathscr{F}$. Now let $\mathscr{D}=\{a, b, c, d\}$. Then there is a one-to-one correspondance $\phi: \mathscr{D} \rightarrow \mathscr{F}$ given by $\phi(a)=F_{a}=X, \phi(b)=$ $F_{b}=\{1,3\}, \phi(c)=F_{c}=\{1,2\}$ and $\phi(d)=F_{d}=\{1\}$. Let $\lambda: \mathscr{D} \rightarrow X$ be the directed net so obtained such that $\lambda(i) \in F_{i}$, for $i=a, b, c, d$. Suppose $\lambda=\{1,2,1,1\}$. Let $I(\lambda)=\{\emptyset,\{a\},\{c\},\{a, c\}\}$. We can see that for $x=1$ and $U=\{1\}$, $\{t \in \mathscr{D}: \lambda(t) \notin\{1\}\}=\{b\} \notin I(\lambda)$. Therefore, $I(\lambda)-\lim \lambda \neq 1$.

\section{Acknowledgement}

Rohini Jamwal would like to thank the University Grant Commission (UGC), New Delhi, India for granting permission for pursuing Ph.D under Faculty Improvement Programme (FIP).

\section{Competing interests}

The authors declare that they have no competing interests.

\section{Authors' contributions}

All authors have contributed to all parts of the article. All authors read and approved the final manuscript. 


\section{References}

[1] V. Baláž, J. Červeńanský, P. Kostyrko, T. Šalát, I-convergence and I-continuity of real functions, Faculty of Natural Sciences, Constantine the Philosoper University, Nitra, Acta Mathematical 5, 43-50, 2002.

[2] N. Bourbaki, General Topology, Part (I) (transl.), Addison- Wesley, Reading (1966).

[3] K. Demirci, I-limit superior and limit inferior, Math. Commun. 6 (2001), 165-172.

[4] H. Fast, sur la convergence statistique, colloq. Math. 2 (1951), 241-244.

[5] H. Halberstem, K. F. Roth, Sequences, Springer, New York, 1993.

[6] D. S. Jamwal, R. Jamwal, S. Sharma, I-convergence of filters, New Trends in Mathematical Sciences, 2016,(accepted).

[7] P. Kostyrko, T.Šalát, W. Wilczynski, I-convergence, Real Analysis, Exch. 26 (2) (2000/2001), 669-685.

[8] P. Kostyrko, M. Mačaj, T.Šalát, M. Sleziak, I-convergence and extremal I-limit points, Math. Slovaca, 55 (4) (2005), $443-464$.

[9] B. K. Lahiri, P. Das, Further results on I-limit superior and I-limit inferior, Math. Commun.,8 (2003), 151-156.

[10] B. K. Lahiri, P. Das, I and I*-convergence in topological spaces, Math. Bohemica, 130 (2) (2005), 153-160.

[11] B. K. Lahiri, P. Das, I and I*-convergence of nets, Real Analysis Exchange, 33 (2) (2007/2008), 431-442.

[12] M. Mačaj, T.Šalát, Statistical convergence of subsequences of a given sequence, Math. Bohemica, 126 (2001), 191-208.

[13] M. Mursaleen and A. Alotaibi, On I-convergence in random 2-normed spaces, Math. Slovaca, 61(6) (2011) 933-940.

[14] M. Mursaleen and S. A. Mohiuddine, On ideal convergence of double sequences in probabilistic normed spaces, Math. Reports, 12(62)(4) (2010) 359-371.

[15] M. Mursaleen and S. A. Mohiuddine, On ideal convergence in probabilistic normed spaces, Math. Slovaca, 62(1) (2012) 49-62.

[16] M. Mursaleen, S. A. Mohiuddine and O. H. H. Edely, On the ideal convergence of double sequences in intuitionistic fuzzy normed spaces, Comput. Math. Appl., 59 (2010) 603-611.

[17] I. Niven, H. S. Zuckerman, An introduction to the theory of numbers, 4th Ed., John Wiley, New York, 1980.

[18] T.Šalát, On statistically convergent sequences of real numbers, Mathematical Slovaca, 30 (1980), No. 2, $139-150$.

[19] T.Šalát, B. C. Tripathy, M. Ziman, On I-convergence field, Italian J. of Pure Appl. Math. 17 (2005), 45-54.

[20] A.Sahiner, M. Gürdal, S. Saltan and H. Gunawan, Ideal convergence in 2-normed spaces, Taiwanese J. Math., 11(5) (2007), 1477-1484.

[21] I. J. Schoenberg, The integrability of certain function and related summability methods, Am. Math. Mon. 66 (1959), $361-375$.

[22] S. Willard, General Topology, Addison-Wesley Pub. Co. 1970. 